Simulation and education

\title{
Feasibility of a first responder programme in rural Bangladesh ${ }^{\text {is }}$
}

\author{
Aminur Rahman ${ }^{\mathrm{a}, *}$, Tom Stefan Mecrow ${ }^{\mathrm{a}}$, Saidur Rahman Mashreky ${ }^{\mathrm{b}}$, \\ A.K.M. Fazlur Rahman ${ }^{\mathrm{b}}$, Nahida Nusrat ${ }^{\mathrm{a}}$, Mahruba Khanam ${ }^{\mathrm{a}}$, \\ Justin Scarr ${ }^{\mathrm{c}}$, Michael Linnan ${ }^{\mathrm{d}}$ \\ a International Drowning Research Centre - Bangladesh (IDRC-B), Dhaka, Bangladesh \\ ${ }^{\mathrm{b}}$ Centre for Injury Prevention Research, Bangladesh (CIPRB), Dhaka, Bangladesh \\ ${ }^{\mathrm{c}}$ Royal Life Saving Society Australia (RLSSA), Sydney, Australia \\ d The Alliance for Safe Children (TASC), Atlanta, USA
}

\section{A R T I C L E I N F O}

\section{Article history:}

Received 5 November 2013

Received in revised form 15 April 2014

Accepted 20 April 2014

\section{Keywords:}

Drowning

CPR training

Resuscitation

Low literacy setting

Rural Bangladesh

LMICs

\begin{abstract}
A B S T R A C T
Objectives: To develop and implement a first responder training programme, assess the feasibility of training lay persons with low literacy in rural Bangladesh and determine the acceptability of the programme in the community.

Methods: A first responder training programme including cardiopulmonary resuscitation (CPR) was developed covering 20 villages in a rural sub-district in north-central Bangladesh. 2398 participants received training and 2120 graduated over a 14-month period. Responders were a mix of adolescents, community volunteers and community elders. The programme was evaluated through post-training assessment of knowledge and skills of participants and performance evaluation of trainers. A focus group discussion was used to assess the response of community leaders to usefulness and community acceptance of the programme.

Results: Materials developed for training include a low-literacy training manual, posters and a training video. Almost 90 per cent (88.4) of participants qualified in post training assessment. Adolescents and community volunteers had higher pass rates than community elders. In all, CPR skills showed a significant decline over 9 months of assessment, while first aid knowledge appeared stable over the same period. Community leaders considered the programme useful for the community and expressed their support for the programme.

Conclusion: Developing a first responder training programme that includes CPR in a rural Bangladesh community is feasible if participants have secondary school attainment. Adolescents and young adults are suitable candidates. Evaluation is ongoing to see whether the programme graduates were able to reduce morbidity and mortality through effective first response efforts.
\end{abstract}

(c) 2014 Elsevier Ireland Ltd. All rights reserved.

\section{Introduction}

Drowning is the leading cause of death in children aged 1-17 in Bangladesh. ${ }^{1}$ Several factors contribute: rural houses lack piped water and have ponds very close to the home, children come

\footnotetext{
A Spanish translated version of the summary of this article appears as Appendix in the final online version at http://dx.doi.org/10.1016/j.resuscitation.2014.04.022. * Corresponding author at: International Drowning Research Centre - Bangladesh (IDRC-B), House - B 162, Road - 23, New DOHS, Mohakhali, Dhaka 1206, Bangladesh.

E-mail addresses: aminur@ciprb.org, aminur61@hotmail.com (A. Rahman), thomasmecrow@yahoo.co.uk (T.S. Mecrow), mashreky@ciprb.org

(S.R. Mashreky), fazlur@ciprb.org (A.K.M.F. Rahman),nusrat_160@hotmail.com (N. Nusrat), kmahruba@yahoo.com (M. Khanam), jscarr@rlssa.org.au (J. Scarr), mlinnan@tasc-gcipf.org (M. Linnan).
}

into daily contact with the pond from earliest childhood, large family sizes result in sibling supervision, and siblings and others lack training in rescue and resuscitation. ${ }^{2}$ Additionally, research has shown rural residents commonly perform traditional, harmful practices for drowning resuscitation, e.g. whirling the victim overhead, thrusting on the stomach and packing them in ashes. . $^{3,4}$ In summary, the incidence of child drowning is high and victims almost never receive resuscitation as bystanders do not have CPR skills and emergency medical systems do not exist in rural areas.

In response to this situation, the International Drowning Research Centre-Bangladesh (IDRC-B) is conducting drowning intervention research. One area is primary prevention of drowning through increased supervision for young children by creating village crèches. Another is secondary prevention of drowning through teaching survival swimming beginning at age 4 . A third is tertiary 
prevention through provision of rescue and resuscitation skills to adolescents and adults in the rural community.

This study describes one activity in the tertiary prevention research; the development and implementation of a first response training programme in rural Bangladesh that includes CPR. It presents evidence for feasibility of training lay persons with low literacy in resource-poor, rural communities and presents results of the community acceptance of the programme.

\section{Methods}

\subsection{Study setting}

The training programme was conducted in Raiganj, a rural subdistrict of Sirajganj in north-central Bangladesh with a drowning incidence of $29.6 / 100,000$ children under 18 years. ${ }^{5}$ The population of the 20 village study area is 45,000 with 45 percent children under 18 years.

\subsection{Study design}

The programme was developed by reviewing first responder training programmes in high income countries (HICs) and other low and middle income countries (LMICs). While a major focus of the programme was drowning response, it included basic first aid for common injuries in addition to CPR. Low-literacy training materials were developed and tested. The testing included effectiveness of the materials and validity of assessment instruments. The training manual developed listed harmful customary practices, discouraged their use and taught correct practices to use in their place. The CPR portion was based on the European Resuscitation Council protocol. ${ }^{6}$ It was chosen as previous research showed a large majority of resuscitation would be done for child drowning. The study was approved by the Centre for Injury Prevention and Research, Bangladesh Ethical Review Committee.

Training used training of trainer's methodology. One male and three female trainers were recruited. Two were nurses and two were non-medical staff with a social science background. After one week of intensive training in theory and practice the trainers conducted a series of two day training courses with two trainers jointly training single sex classes of 15 participants. Training was minimally didactic using mostly hands-on training methods. Adult manikins were used for both adult and child CPR training as paediatric manikins were not available. Locally available resources (e.g. gauze, bandage, and sticks for splints) were used for other first aid skills.

Participants were village residents purposefully selected for educational attainment or social status. Trainees were adolescents attending secondary school, and adults of stature in the village, such as mothers who hosted the village crèche, village swimming instructors, and village elders. The number of trainees selected provided a ratio of one first responder to every 20 community residents. Training occurred between March 2011 and April 2012. Graduates were awarded a certificate and identity card as first responders. Retention of CPR and first aid knowledge and skills was tested on different samples of randomly selected graduates at one, three, six, and nine months after initial training. Instrumented testing manikins are not available in Bangladesh. Therefore, skills such as ventilation were assessed visually for effectiveness rather than measured quantitatively; likewise chest compressions were assessed visually for depth, force and tempo without instrumentderived measurement data.

A structured checklist for the key skills and knowledge of CPR and first aid was developed by modifying examples in the CPR and first aid training literature. The process used local experts on
CPR, first aid and staff with a background in development of teaching materials for Bangladesh. Further checklists were developed to assess trainers' rapport building capacity with the trainees, communication skills, use of training aids, knowledge and skills transfer capacity and assessment skills. Trainers' performances were evaluated by observation of a sample of 16 sessions, four for each trainer during the course of the training.

Community acceptance was determined by a focus group. Dimensions relating to acceptance and satisfaction were included based on previous assessments of village programmes. A script ensured all items were covered. Discussions were in Bangla and assessment was done by native Bangla speaking research staff at IDRC-B. The focus group discussion (FGD) was conducted during February 2012 with nine community leaders to explore their responses to the training programme. The FGD was audio-taped with the respondents' permission.

\subsection{Data analysis and assessment}

Frequency distributions of initial assessment and reassessments were compared to assess retention of knowledge and skills. Chi square for trend was used to assess whether the decline in measured scores for skills and knowledge was significant. Trainers' performance was assessed by observation using the checklist. A transcript of the FGD was prepared and analyzed to identify ways the participants responded to various issues about the training programme.

\section{Results}

A total of 2440 villagers were invited to participate in the programme. Forty seven (1.9 per cent) refused, and 2398 participants were trained during the 14 month period. Similar proportions of males ( 48.6 per cent) and females ( 51.4 per cent) participated. Adolescents (10-17 years) of both sexes were 59.8 per cent of graduates with the remaining 40.2 per cent adults (18+ years).

Trainees included adolescents attending school (10-17 years $n=1150$, male $=50.3$ per cent, female $=49.7$ per cent $)$, adolescent not attending school (10-17 years $n=283$, male $=35.3$ per cent, female $=64.7$ per cent $)$, adult community volunteers $(n=587$, male $=47$ per cent, female $=53$ per cent) $($ medicine shopkeepers, mothers hosting village crèches, community swimming instructors) and adult community leaders and elders ( $n=378$, male $=55.6$ per cent, female $=44.4$ per cent $)$ (school teachers, religious leaders, members of the local and village government).

Of 2398 participants who began training, 2120 (88.4 per cent) passed and were given graduation certificates, 256 (10.7 per cent) failed to qualify and 22 ( 0.9 per cent) dropped out. Among all male and female participants 91.8 per cent and 85.2 per cent respectively passed the final assessment. Passing rates in adolescent school attendees and non-attendees, and community volunteers were higher than community leaders. The lowest passing rate (76.2 percent) was among female community leaders (Fig. 1).

\subsection{Sex}

A higher proportion of males than females passed in each category. This was significant in adolescent school attendees $\left(\mathrm{Chi}^{2}=16.02, p=<0.05\right)$, adolescent non-school going attendees $\left(\mathrm{Chi}^{2}=4.18, p=0.04\right)$ and community leaders $\left(\mathrm{Chi}^{2}=4.45, p=0.03\right)$. There was no significant difference between male and female community volunteers $\left(\mathrm{Chi}^{2}=2.77, p=0.1\right)$.

\subsection{Education}

Current school attendance made no significant difference to pass rates in either adolescent males $\left(\mathrm{Chi}^{2}=2.30, p=0.32\right)$ or adolescent 


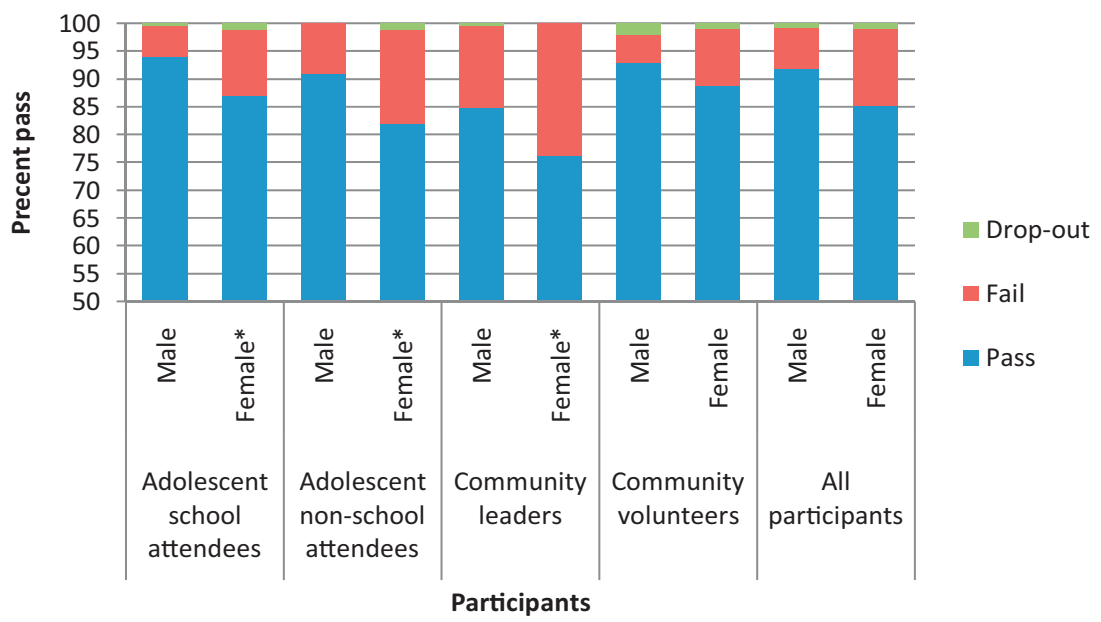

Fig. 1. Distribution of participants by category, sex and assessment result. ${ }^{*} p<0.05$ for difference between males and females.

females $\left(\mathrm{Chi}^{2}=3.33, \mathrm{p}=0.19\right)$. However, amongst all participants there was a positive association between the numbers of years of education of a participant and their pass rate (Pearson's $R=0.150$, $p=<0.05$ ).

\subsection{Age}

Pass rates between adolescents (10-17 years) and adults (18+ years) differed significantly. The adolescent pass rate was 89.5 per cent and the adult pass rate was 86.8 per cent $\left(\mathrm{Chi}^{2}=3.90, p=0.048\right)$.

\subsection{Retention of knowledge and skills of first responders}

Retesting was done at one, three, six and nine months on 182, 450, 461 and 409 participants respectively. The instrument used had a Chronbach alpha of $0.77,0.79$ and 0.91 for the three skills-based portions of CPR assessment and 0.58 for the first aid knowledge assessment.

For those retested one month after initial training, correct performance was uniformly high (89.6-98.4 per cent) except action for vomiting (83.0 per cent). However, most skills showed a decline at three months that continued at six and nine months. The trend was significant for all skills tested and decreases ranged from 8.9 per cent (check for danger) to 40 per cent (action for vomiting). The average decline in skill was 15.3 per cent over the 9 months. In contrast, knowledge items had uniformly high assessment at each time of assessment. While there was a trend for a decline across the 9 months of testing, it was not significant. The average decline in knowledge was 5.4 per cent over the 9 months (Table 1 ).

\subsection{Community leaders' response to the first responder training programme}

A FGD explored the community leaders' response to the programme.

\subsubsection{Existing community practice during emergencies}

Usual practice for resuscitation after drowning was to spin the victim overhead or to press on the stomach to expel water. To treat a fracture or dislocation the injured part is shaken and the victim taken to traditional healers. For a cut injury crushed or chewed leaves or grass are applied to the wound. Treatment for animal bites involves giving food items that have been 'enchanted' especially salt. Common practices for treating burns are applying raw egg, mud, chilli or turmeric paste and coconut oil.

\subsubsection{Opinion about the training programme}

All nine FGD respondents expressed support for the programme. A female teacher commented

"In school text books there are topics on first aid but those are only theoretical knowledge; but now we understand skills need to be taught. ... Even we (the teachers) never received such skill based training. ....I understand it is not difficult, anybody can learn these skills."

\subsubsection{Support to implement the training programme}

Community leaders said they would recommend community volunteers participate in the training programme and they would ask community people to avoid harmful practices and receive correct care from the trained first responders. The leaders also expressed willingness to protect the first responder if there was a threat of reprisal occurring after first aid given.

\subsubsection{Scaling-up of the first responder training programme}

Teachers noted that this training should be included in the regular school curriculum. They suggested grade 6-10 students are old enough to learn the skills.

\section{Discussion}

We present the initial results of a village-based first response system in rural Bangladesh designed to be effective in training rural residents with relatively low educational attainment and to be appropriate for common injuries and other emergencies normally encountered. Training materials were designed to be used in low-literacy settings and taught using hands-on, visually guided methods with minimal use of didactic methods. Lack of instrumented manikins and other higher technology training aids required adaptations in both training and assessment. Trainees' knowledge and skills were evaluated at graduation and intervals of one, three, six and nine months. A significant decline was found in all CPR skills tested. Key members of the village community felt the training was useful and needed. Clinical outcomes were not part of the evaluation process and will be reported on in future.

Evidence on effective first response programmes that include CPR training is almost entirely from HICs where participants are literate, CPR is commonly seen in media shows and often is introduced within formal schooling systems. ${ }^{7,8}$ In Bangladesh, education levels are comparatively low with a large proportion of the population illiterate. The literacy rate in rural Bangladesh among 15 years and 
Table 1

Results of testing retention of skills of CPR and knowledge on first aid by group tested.

\begin{tabular}{|c|c|c|c|c|c|c|c|}
\hline \multirow[t]{2}{*}{ Skills and knowledge re-tested ${ }^{\mathrm{a}}$} & & \multicolumn{4}{|c|}{ Time interval after initial assessment ${ }^{b}$} & \multicolumn{2}{|l|}{ Statistics } \\
\hline & & $\begin{array}{l}1 \text { month } \\
N=182\end{array}$ & $\begin{array}{l}3 \text { months } \\
N=450\end{array}$ & $\begin{array}{l}6 \text { months } \\
N=461\end{array}$ & $\begin{array}{l}9 \text { months } \\
N=409\end{array}$ & $\begin{array}{l}\text { Chi square for } \\
\text { trend }\end{array}$ & $P$ value \\
\hline \multicolumn{8}{|c|}{ Per cent able to demonstrate first response to an unconscious casualty } \\
\hline 1 & Check for dangers & 98.4 & 96.9 & 94.4 & 89.5 & 27.5 & $<0.01$ \\
\hline 2 & Check for response & 96.2 & 95.6 & 91.8 & 88.3 & 19.9 & $<0.01$ \\
\hline 3 & Shout/Call for help & 97.8 & 96.0 & 93.7 & 82.6 & 57.5 & $<0.01$ \\
\hline 4 & Open the airway & 91.2 & 78.0 & 76.8 & 63.8 & 48.1 & $<0.01$ \\
\hline 5 & $\begin{array}{l}\text { Check for normal } \\
\text { breathing }\end{array}$ & 91.8 & 79.8 & 77.0 & 65.0 & 50.6 & $<0.01$ \\
\hline 6 & $\begin{array}{l}\text { Demonstrate action for } \\
\text { vomiting }\end{array}$ & 83.0 & 58.4 & 66.8 & 43.0 & 57.1 & $<0.01$ \\
\hline 7 & $\begin{array}{l}\text { Turn into the recovery } \\
\text { position }\end{array}$ & 89.0 & 78.0 & 81.3 & 68.0 & 25.7 & $<0.01$ \\
\hline \multicolumn{8}{|c|}{ Per cent able to demonstrate first response to an unconscious non breathing casualty } \\
\hline 1 & Check for dangers & 98.4 & 96.4 & 94.4 & 89.2 & 26.3 & $<0.01$ \\
\hline 2 & Check for response & 95.6 & 93.1 & 91.3 & 88.0 & 11.5 & $<0.01$ \\
\hline 3 & Shout/Call for help & 90.7 & 93.1 & 92.0 & 81.2 & 39.0 & $<0.01$ \\
\hline 4 & Open the airway & 89.6 & 78.2 & 79.6 & 61.4 & 56.5 & $<0.01$ \\
\hline 5 & $\begin{array}{l}\text { Check for normal } \\
\text { breathing }\end{array}$ & 89.6 & 78.0 & 75.3 & 65.8 & 37.4 & $<0.01$ \\
\hline 6 & Give 5 rescue breaths & 90.7 & 85.3 & 84.2 & 74.6 & 26.1 & $<0.01$ \\
\hline 7 & $\begin{array}{l}\text { Perform } 30 \text { chest } \\
\text { compression }\end{array}$ & 96.2 & 90.0 & 86.8 & 79.7 & 34.8 & $<0.01$ \\
\hline 8 & $\begin{array}{l}\text { Continue } 30 \\
\text { compressions followed } \\
\text { by } 2 \text { rescue breaths for } \\
\text { a period of } 2 \text { min }\end{array}$ & 96.2 & 86.9 & 86.8 & 78.0 & 30.0 & $<0.01$ \\
\hline \multicolumn{8}{|c|}{ Per cent able to demonstrate chest compressions and rescue breathing on an unconscious child } \\
\hline 1 & $\begin{array}{l}\text { Perform } 30 \text { chest } \\
\text { compressions }\end{array}$ & 98.9 & 88.7 & 88.3 & 85.3 & 15.2 & $<0.01$ \\
\hline 2 & $\begin{array}{l}\text { Continue } 30 \\
\text { compressions with } 2 \\
\text { rescue breaths for a } \\
\text { period of } 2 \text { min (child) }\end{array}$ & 98.4 & 89.1 & 87.2 & 83.9 & 20.7 & $<0.01$ \\
\hline \multicolumn{8}{|c|}{ Per cent able to correctly answer knowledge of first aid } \\
\hline 1 & Water rescue & 100.0 & 97.6 & 98.3 & 97.3 & 1.9 & 0.17 \\
\hline 2 & $\begin{array}{l}\text { Wound injury with } \\
\text { bleeding }\end{array}$ & 99.5 & 88.7 & 96.3 & 95.8 & 3.7 & 0.055 \\
\hline 3 & Burn injury & 100.0 & 97.3 & 98.7 & 98.8 & 0.2 & 0.69 \\
\hline 4 & Animal bite & 98.4 & 91.8 & 94.4 & 93.2 & 0.8 & 0.38 \\
\hline 5 & Casualty transportation & 93.4 & 88.0 & 91.3 & 88.5 & 0.6 & 0.45 \\
\hline
\end{tabular}

a When assessed at the end of the course, $100 \%$ of participants were able to demonstrate the skill or knowledge.

b Samples were independently selected at each interval rather than the same sample followed longitudinally, causing the variation seen between sample intervals.

over is 46 per cent. ${ }^{9}$ In addition to low literacy, rural people lack familiarity with basic first aid. ${ }^{3}$

This study provides evidence that a content-appropriate first aid course that includes CPR can be developed to train lay persons in rural Bangladesh. However, doing so required substantial modifications compared to HICs. Modifications included issues of literacy, suitability of teaching, culture-specific issues and medicolegal issues.

Teaching modifications included development of a manual and visual teaching aids effective for the low literacy level. The manual used extensive visual graphics so trainees could understand the basic concepts of CPR and first aid. The training process used hands-on demonstrations and video tutorials. Training was done in single-sex classes due to strong community preference. Community leaders participated and took active roles to increase programme support in the community and give leadership support for responders. Other modifications included having credible authority figures present information on common knowledge and practices current among laypeople that are harmful when used.

In developing the programme two key challenges were encountered, namely cessation of CPR and the safety and potential for harm to first responders. Most CPR programmes in HICs assume CPR will continue until trained medical personnel arrive and make the decision to stop resuscitation. Emergency medical services are not present in rural areas so it was necessary to provide a formal cessation step in the CPR protocol. In order to give it credibility in social and medico-legal terms, a working group of six Bangladeshi medical and surgical specialists mandated a combination of chest compressions and mouth-to-mouth ventilation as the preferred method of resuscitation. Specific cessation guidelines stated responders should consider stopping after $30 \mathrm{~min}$, if the resuscitator is physically exhausted, or if professional medical assistance arrives.

The development of guidelines by nationally credible experts was an effort to provide formal legal and informal social protection for responders. Bangladesh, like most LMICs lacks Good Samaritan laws common in HICs. A responder could be deemed to commit a harmful act in stopping resuscitation on their own. Provision of cessation guidelines by a professional working group provided legal protection for this. Bangladesh also lacks a tradition of social acceptance for voluntarism. In fact, for those who are not government officials and who respond to emergencies, there is threat of reprisal especially in rural areas. In these areas, laws are often enforced immediately by vigilante groups that form spontaneously when witnessing an incident or in reprisal attacks following. People commonly flee the scene of a serious incident due to fear of reprisal. Encouraging a lay person to provide first aid or CPR could place them in danger of being attacked, particularly in the result of a death at the scene of the accident. Potential for this was reduced by providing expert guidelines to follow, including 
community leaders and authority figures as first responders in the programme, and holding extensive community awareness meetings in the areas served by the first responders.

While there were higher pass rates for males compared to females in some groups as well as between adolescents and adults, the differences are not sufficient to preclude either group from being considered as participants in future first response programmes.

Importantly, retention of CPR and first aid knowledge and skills may be a significant obstacle to maintain an effective first response network in Bangladesh. In HICs, CPR skills have been shown to deteriorate after 30 days following certification and continued regular training is required to ensure skill levels are retained. ${ }^{10,11}$ In this study, there was decline in key CPR skills after the one month assessment which continued in the ensuing eight months of evaluation. The drop off in competency was statistically significant. Knowledge related to first aid practices appeared to be more durable over the 9 month time period. Retention length may be a key factor in feasibility because the low resource environment precludes refresher training at any interval less than one year and possibly longer. Whether the drop off in skills tested compromises the effectiveness of the programme will be an important question answered through clinical outcome evaluation.

The programme was designed with practicality in teaching CPR. The focus was on what skills were fundamental to ensure effective CPR and less so on perfect adherence to the protocol. For example, providing chest compression and ventilation was prioritized over calling for help and/or checking for danger to the rescuer. The clinical outcome evaluation is being conducted in the same practical manner. If programme graduates are able to conduct CPR resulting in successful resuscitation of victims without respiration or pulse, the CPR portion of the first response programme will be judged as effective. Follow-up on all CPR events is ongoing and will be reported in future.

It is important to note that the trainees were selected for willingness to attend the course. This likely also selected for factors such as willingness to learn and openness to new things, which may not be common in a rural community. Thus, findings may not be generalizable to the broader rural population.

Other limitations relate to the low resource environment. The curricula for both first aid and CPR were simplified for teaching for ease of learning and the environment to be used in. The curriculum currently lacks evaluation of effectiveness and without instrumented manikins and other training aides there is no quantitative data on skills effectiveness. Also by nature as a first effort, a baseline is lacking for evaluation of the programme itself in Bangladesh; without similar activities in other low and middle income countries, there is not a means for comparison with such efforts. None-the-less, the pilot was conducted to see if it is possible to create a needed first response programme in a rural village in a low-income setting.

\section{Conclusion}

Developing a first responder training programme which includes CPR in a rural community with low literacy appears feasible. Adolescents and adults with secondary education are suitable candidates for first responders. Inclusion of high-status village residents increases community acceptance and should address safety issues for responders. Research is ongoing to evaluate clinical outcomes and provide evidence whether graduates reduce morbidity and mortality through effective first response.

\section{Conflicts of interest statement}

None.

\section{Acknowledgements}

We thank the Australian Government Agency for International Development (AusAID) for funding the project, and Royal Life Saving Society Australia (RLSSA) and The Alliance for Safe Children (TASC) for their technical support. We gratefully acknowledge the local government officials, community leaders and the community people of Raiganj who participated in the training programme. We thank Laerdal Australia for providing the manikins.

Additional note: Materials developed are available on request. Final written materials are in Bangla, but near-final English drafts are available. The training video is in Bangla.

\section{References}

1. Linnan M, Anh LV, Cuong PV, et al. Child mortality and injury in Asia: Survey results and evidence, Working Paper 2007-05, Special Series on Child Injury No. 2. Florence: UNICEF Innocenti Research Centre; 2007.

2. Linnan M, Rahman A, Scarr J, et al. Child Drowning: Evidence for a newly recognized cause of child mortality in low and middle income countries in Asia Working Paper 2012-07, Special Series on Child Injury No.2. Florence: UNICEF Office of Research; 2012.

3. Rahman A, Rahman F, Shafinaz S, Linnan M. Bangladesh Health and Injury Survey: report on children. Dhaka: Directorate General of Health Services, Ministry of Health and Family Welfare, Govt. of the People's Republic of Bangladesh; 2005

4. Borse NN, Hyder AA, Streatfield PK, Arifeen SE, Bishai D. Childhood drowning and traditional rescue measures: case study from Matlab, Bangladesh. Arch Dis Child 2011;96:675-80, http://dx.doi.org/10.1136/adc.2010.202010.

5. CIPRB. Injury Surveillance Report on the PRECISE Project. CIPRB; 2011.

6. Nolan P. Resuscitation guidelines. London, UK: Resuscitation Council; 2010.

7. Diem SJ, Lantos JD, Tulsky JA. Cardiopulmonary resuscitation on television miracles and misinformation. N Engl J Med 1996;334:1578-82.

8. Kanstad BK, Nilsen SA, Fredrikse K. CPR knowledge and attitude to performing bystander CPR among secondary school students in Norway. Resuscitation 2011;82:1053-9.

9. Bangladesh Bureau of Statistics. Bangladesh Literacy Assessment Survey 2008 Dhaka: Bangladesh Bureau of Statistics; 2008.

10. Anderson GS, Gaetz M, Masse J. First aid skill retention of first aid attendants within the workplace. Scand J Trauma Resusc Emerg Med 2011;19:11.

11. Sutton RM, Niles D, Meaney PA, et al. Low-dose, high-frequency CPR training improves skill retention of in-hospital pediatric providers. Pediatrics 2011;128:e145-51, http://dx.doi.org/10.1542/peds.2010-2105. 